\title{
Kontinuierliche Messung der Assimilation und Atmung mariner Algen mittels der elektrochemischen Sauerstoffbestimmung
}

\author{
W INFRID SCHRAMM \\ Institut für Meereskunde der Universität Kiel (Botanische Abteilung)
}

\begin{abstract}
Continuous measurement of assimilation and respiration of marine algae, employing electro-chemical oxygen determination. An apparatus is described for the continuous measurement of assimilation and respiration of marine organisms, employing the principle of electro-chemical oxygen determination. The measurement of $\mathrm{O}_{2}$ takes place in a closed system with a stabilized membrane-covered Pt-electrode (Clark-principle), having the following properties: The electrode gives $90 \%$ of the full response in 14 to 16 seconds; drift over a period of a few days amounts to about $0.8 \%$, over a few weeks, to about $2 \%$. In the range from 0 to $10 \mathrm{mg} \mathrm{O}_{2} / \mathrm{l}$, the relationship between the depolarization current and $\mathrm{O}_{2-}$ concentration is linear. The calibration curve goes through the origin. With the described assembly, variations in oxygen changes down to $0.0035 \mathrm{mg}$ can be detected. An example of the usefulncss of the new method is shown on the basis of assimilation and respiration measure ments on the alga Fucus vesiculosus $L$.
\end{abstract}

\section{ALLGEMEINES}

Die gebräuchlichste Methode zur Bestimmung des molekular in Seewasser gelösten Sauerstoffs ist das von WINKLER (1888) beschriebene Verfahren. Bis heute kann auf diese einfache und exalste Methode bei der Untersuchung des Gaswechscls mariner Organismen nicht verzichtet werden. Ein wesentlicher Nachteil besteht allerdings darin, daß auf diese Weise besonders kurzfristige Veränderungen in der Menge des gelösten Sauerstoffs nicht kontinuierlich erfaßt werden können.

Für eigene Untersuchungen zur Austrocknungs- und Temperaturresistenz mariner Algen ${ }^{1}$ waren bei der Wahl einer geeigneten Methode neben der Forderung nach der Kontinuität der Messung vor allem folgende Gesichtspunkte maßgebend: (1) Spezifität der Messung für O; (2) Messung in Meerwasser; (3) Hohe Empfindlichkeit und großes zeitliches Auflösevermögen; (4) Registrierbarkeit mittels Schreiber; (5) Geringe Anzeigeverzögerung; (6) Technische Eignung für Serienuntersuchungen.

Die im Rahmen einer Dissertation durchgeführten Untersudungen stehen vor ihrem 
Unter den bisher gebräuchlichen, kontinuierlich arbeitenden Möglichkeiten (EgLE 1960, Simonis 1960, Müller 1958) erschien uns das Verfahren zur elektrochemischen $\mathrm{O}_{2}$-Bestimmung für die geplanten speziellen Untersuchungen am geeignetsten.

Das außerordentlich genau arbeitende polarographische Verfahren zur Sauerstoffbestimmung in Elektrolyten mittels der von HeyrovsKY (1922) eingeführten $\mathrm{Hg}$ Tropfelektrode hat den Nachteil, daß es sehr empfindlich gegen Erschütterungen ist. Außerdem besteht die Gefahr, daß das Untersuchungsobjekt mit dem Quecksilber der Tropfelektrode in Berïhrung kommt.

Tö̀t $(1929,1952,1958)$ und seine Mitarbeiter sowie OHLe (1953) und andere verwendete Anordnungen, bei denen nach dem Prinzip der inneren Elektrolyse eine Edelmetallelektrode durch den im Versuchswasser gelösten Sauerstoff depolarisiert wird. Für zahlreiche biologische Untersuchungen hat sich vor allem dieses Verfahren durchgesetzt und vielfach bewährt (Zusammenfassungen bei TöDT 1958, MüLlER 1958). Allerdings weisen diese Meßeinrichtungen (z. B. TöD'T 1958, „Bioflux") erhebliche Nachteile auf. Die frei in der zu messenden Lösung stehenden Elektroden reagieren außerordentlich empfindlich auf Anderungen der Turbulenzverhältnisse. Weiter ändert sich mit verschiedenem Elektrolytgehalt die Leitfähigkeit und damit der Depolarisationsstrom (OHLе 1953, Ambürul 1958). Bei Messungen im Meerwasser belegt sich die Kathode außerdem mit Spurenmetallen und Erdalkali-Hydroxyden beziehungsweise -Karbonaten. Die Folge davon ist ein unkontrollierbares Absinken des Depolarisationsstromes (Grasshoff 1962b).

Für Untersudhungen, wie sie hier geplant waren, erwiesen sich diese Verfahren also als nicht geeignet. Es wurde daher eine von ClarK (1953) angegebene, von KaNWISHER (1959) zuerst für biologische Untersuchungen angewendete und von GLEICHMANN \& LüBBERs (1960) verbesserte Meßanordnung verwendet, bei der das Elektrodensystem und der Elektrolyt von dem zu messenden Medium durch eine dünne Membran elektrisch abgetrennt wird. Der Elektrodenaufbau wurde von Grasshoff (1962b) übernommen und im Verlaufe der Arbeiten für die notwendigen Anforderungen geringfügig abgeändert und verbessert. Die Entwicklung der Apparatur wurde von Herrn Dr. Grasshoff, Kiel, durch wertvolle Ratschläge und Überlassung von Hilfsmitteln großzügig unterstützt, wofür bestens gedankt sei.

Als besonders nachteilig erwies sich auch die in den meisten früheren Anordnungen schwierige Zugänglichkeit des Assimilationsraumes. Bei der Konstruktion der neuen Apparatur wurde daher vor allem Wert gelegt auf die Möglichkeit zur ungehinderten, schnellen Einbringung verschiedenartiger Untersuchungsobjekte.

\section{PRINZIP DER METHODE UND 'THEORET'ISCHE GRUNDLAGEN}

Der polarographische Meßkreis (Abb. 1) besteht aus einer Spannungsquelle (1), mit der eine Spannung an die Meßzelle (2) gelegt werden kann. In der Meßzelle tauchen eine Pt- und eine Ag-Elektrode $(3,4)$ in einen Elektrolyten $(0,2 \mathrm{n} \mathrm{KOH})$ ein (5). Von dem zu messenden Medium ist diese Anordnung durch eine dünne, wasserabstoBende Membran (Lupolen BASF, $20 \mu$ ) getrennt (6). Zwischen dieser Membran und 
der Platinelektrode liegt eine $12 \mu$ starke Cellophanfolie (7). Diese hydrophile Zwischenschicht dient zur Ausbildung einer definierten, elektrolytführenden Zone.

Legt man in diesem Elektrodensystem eine geringe negative Spannung an die $\mathrm{Pt}$ Elektrode und registriert den fließenden Strom, so ergibt sich eine Stromspannungskurve, wie sie in Abbildung 2 dargestellt ist. Ein Teil der aus der zu messenden Lösung durch die Membran hindurchdiffundierenden Sauerstoffmoleküle wird an der

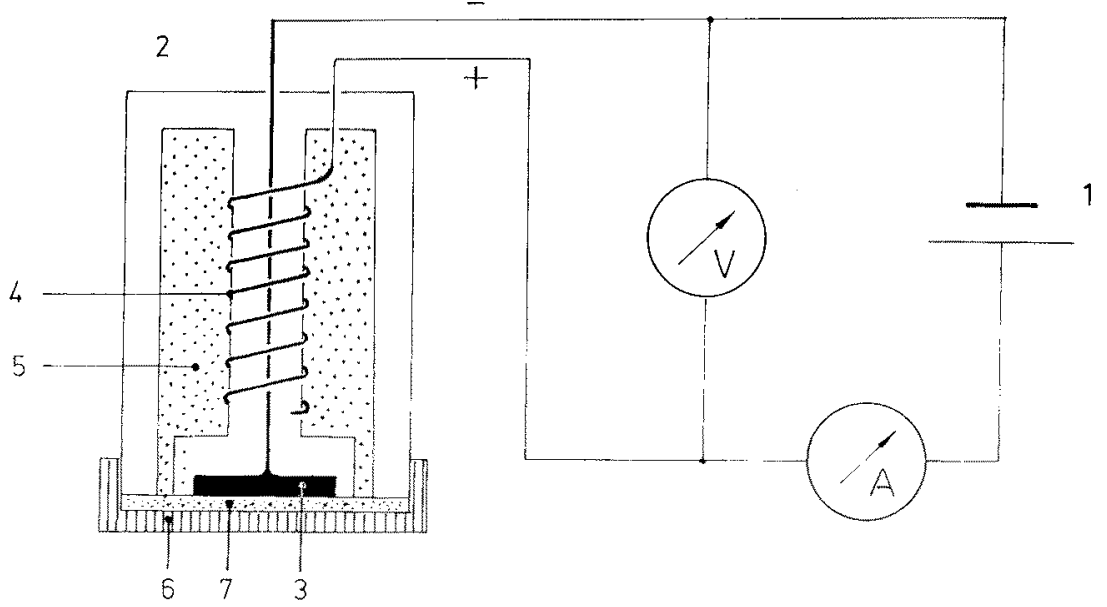

Abb. 1: Schema der MeBanordnung zur elektrohemischen O-Bestimmung. 1 Spannungsquelle, 2 polarographische Meß\%elle, 3 Pt-Kathode, $4 \mathrm{Ag}$-Ago-Anode, 5 Elcktrolytraum, 6 LupolenFolic, 7 Cellophan

polarisierten Platinelektrode durch Anlagerung von Elektronen zu $\mathrm{H}_{2} \mathrm{O}_{2}$, und durch zwci wcitere zu H.O reduziert. Nach Winkelmann (1956) lassen sich die Vorgänge in folgender Form zusammenfassen:

$$
\mathrm{O}_{2}+4 \mathrm{H}^{+}+4 \mathrm{e}=2 \mathrm{H}_{2} \mathrm{O}
$$

Bei Erhöhung der angelegten Spannung steigt dicser Reduktions- oder Diffusionsstrom soweit an (Abb. 2, Bereich a), bis sämtliche an die Elektroden-Oberfläche herandiffundierenden O-Moleküle reduziert werden, das heißt die Sauerstoff-Konzentration ist an der Platimelektrode gleich Null. Eine weitere Lrhöhung der angelegten Spannung hat keinen Einfluß mehr auf die Stromstärke. Der jetzt fließende sogenannte Diffusionsgrenzstrom (Bereich b) erhöht sich nur durch zusätzlich herandiffundierenden Sauerstoff; er ist dem $\mathrm{pO}_{2}$ in der zu messenden Lösung proportional. Erst bei weiterer Erhöhung der Spannung (Bereich c) steigt der Strom weiter an. Es liegen jetzt andere stromliefernde clektrochemische Abläufe vor.

$\mathrm{Da}$ der Depolarisationsstrom diffusionskontrolliert ist, andererseits eine gleichmäßige Heranführung von $O_{2}$-Molekeln aus der weiteren Umgebung der Pt-Elcktrode kaum möglich ist, ist es notwendig, die Diffusionsvorgänge auf eine wohldefinierte Zone zu beschränken. Dies wurde von Gleichmann \& Lübbers (1960) erteicht, indem 
zwischen der zum Außenmedium abgrenzenden Membran und der Pt-Elektrode eine hydrophile, elektrolytführende $Z$ wischenschicht durch Einlegen einer Cellophanfolie geschaffen wurde. Ein Rührmagnet unterhalb der Meßzelle sorgt für eine so große Turbulenz, daß an der Membranaußenfläche stets der gleiche $\mathrm{pO}_{2}$ herrscht wie in dem gesamten Versuchswasser (Gleichmann \& LübBers 1960, Grasshoff 1962b).
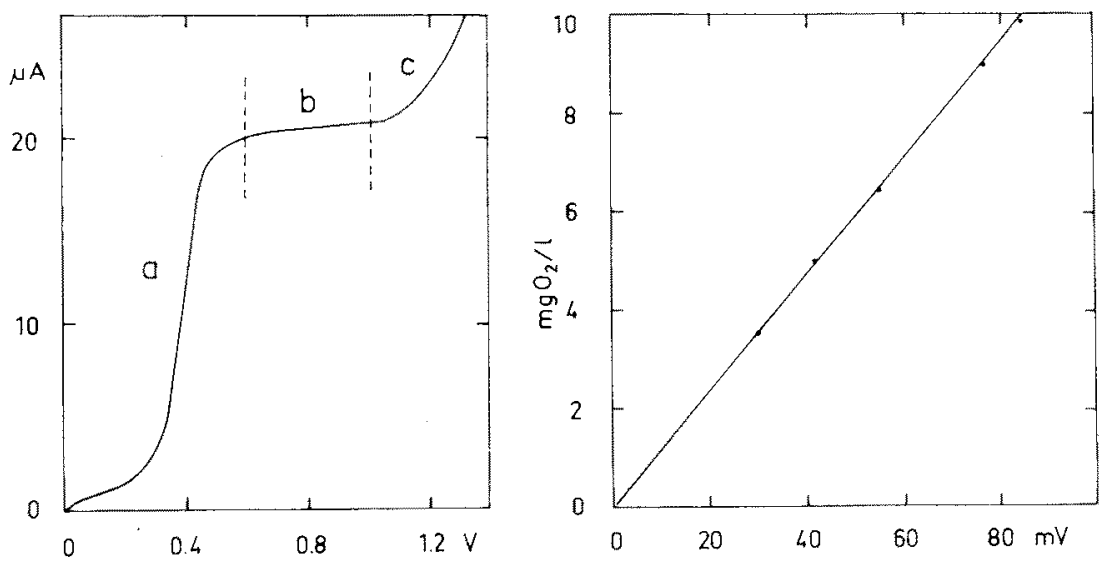

Abb. 2: Polarogramm für Sauerstoff (Luft, $18^{\circ} \mathrm{C}$ )

Abb. 3: Darstellung der Eichkurve für $\mathrm{O}_{2}$ (bei $15^{\circ} \mathrm{C}$, in Seewasser von $15 \% \mathrm{~S}$ )

\section{BESCHREIBUNG DER MESSAPPARATUR}

Aus Grïnden der Meßgenauigkeit wählten wir ein geschlossenes System. Mit Hilfe der polarographischen Meßzelle wird in einem abgeschlossenen Volumen der Zuwachs an photosynthetisch erzeugtem beziehungsweise die Abnahme des durch Atmung verbrauchten Sauerstoffs registriert.

Um ein fehlerfreies und sauberes Arbeiten zu gewährleisten, wurde das eigentliche Kernstück der Meßapparatur, die Assimilationskammer (Abb. 5 A, B), soweit wie möglich aus Glas angefertigt. Die Verwendung von Glas hat folgende Vorteile (siehe auch Gleichmann \& Lübbers 1960): (1) Durchsichtigkeit, (2) chemische Indifferenz, (3) gute Reinhaltungsmöglichkeiten, (4) Unlöslichkeit für Gase, (5) relativ gute Wärme-

Abb. 5 A, B: Assimilation sk a m m e r (A Längsschnitt, B Aufsicht). 1 Elektrodengefäß und Umwälzpumpe, 2 Assimilationszelle, 3 Kühlmantel, 4 Tohldeckel, 5 Druckausgleich, 6 Ablauf, 7 Kontrollthermometer, $8 \mathrm{Zu}$ - und Abflußstutzen, 9 Uberlauf und Abfluß, 10 Objektträger, 11 Weichgummidichtung, 12 Führungsstifte für den Hohldeckel. C: M e Bz ell e (Schnitt). $13 \mathrm{Pt}$-Kathode, 14 Ag-AggO-Anode, 15 Elektrodenträger (Glas), 16 Mantel (PVC), 17 Konushülse (PVC), 18 Schraubventil (PVC), 19 O-Ringdichtung, 20 Elektrolytraum, 21 Rille für die Befestigung der Folien, 22 elcktrische Ableitungen, 23 Kontermutter (PVC), $24 \mathrm{Hg}$-Kontakt. D: Schem a der Versuchsanordnung. 25 Schreiber, 26 Oz-Elektroden-Schaltteil, 27 polarographische Meßzelle, 28 Styropor-Isolierung, 29 Assimilationskammer, 30 Magnetrührer, 31 Temperaturbad, 32 Thermostat, 33 Gegenkühlung, 34 Lichtquelle, 35 Wärmefilter 
A

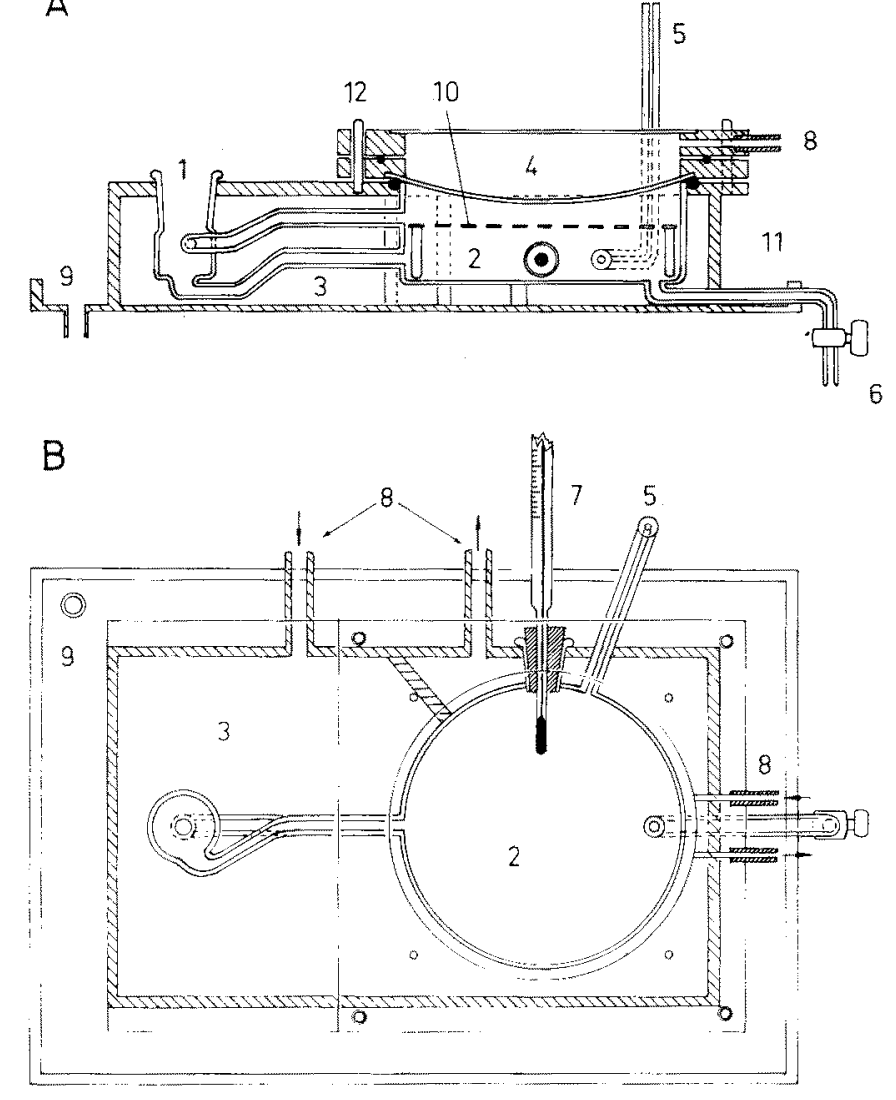

C

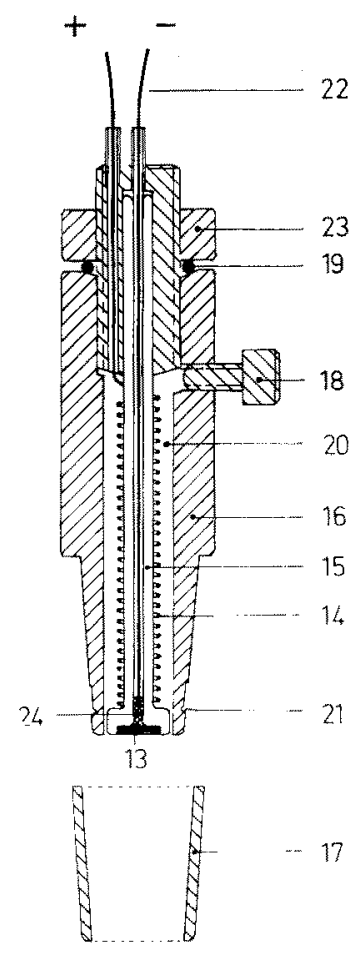

D
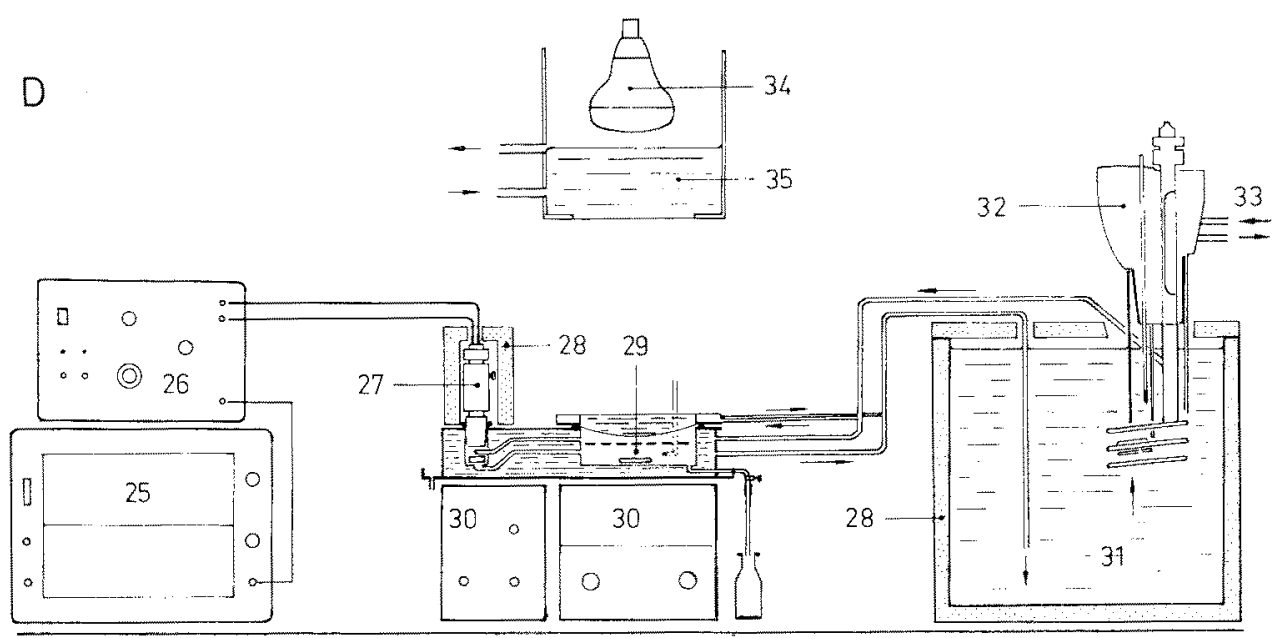
leitfähigkeit. Bei Verwendung von zunächst aus Plexiglas angefertigten Kammern konnten keine brauchbaren Ergebnisse erzielt werden.

Ahnliche Beobachtungen machten auch Severinghaus \& Bradley (1958) und Rooth, Sjöstedt \& Calligara (1960). Je nach Sorte und Alterungsgrad löst sich $\mathrm{O}_{2}$ in Plexiglas in verschieden starkem Maße (BARBEy 1953), so daß unkontrollierbare Meßfehler auftreten können. Außerdem setzen sich besonders leicht Verunreinigungen in Kratzspuren fest, die sich bei häufigem Gebrauch einer aus Plexiglas angefertigten Apparatur nicht vermeiden lassen. Ein weiterer wesentlicher Nachteil liegt in der außerordentlich schlechten Wärmeleitfähigkeit des Plexiglases.

Das zu untersuchende Objekt liegt in einer gläsernen (Jenaer Geräteglas) Assimilationskammer (Abb. $5 \mathrm{~A}[2])(\mathrm{h}=3,3 ; \varnothing=11,0$; Wandstärke $=0,2 \mathrm{~cm}$ ) von $307 \mathrm{ml}$ Inhalt auf einem biegsamen, durchlöcherten Zelluloidträger (Abb. 5 A [10]). Diese Zelle kann mit einem Hohldeckel (4) in kürzester Zeit verschlossen werden. Die Dichtung erfolgt durch einen Weichgummiring (11). Um beim Schließen keine störenden Luftblasen zu erhalten, ist die gläserne Innenseite des Deckels nach außen gewölbt. Der für das Schließen des Deckels notwendige Druckausgleich erfolgt über eine seitlich in die Wandung der Kammer eingesetzte Kapillare (5). In zwei weiteren Bohrungen sind ein Abfluß (6), über den auch die Proben für die Eichung durch Winkler-Bestimmungen abgelassen wurden, sowie ein 14er-Schliff zur Aufnahme eines $1 / 10^{\circ}$-CKontrollthermometers (7) eingekittet. Da sich bei ersten Versuchen ergab, daß bei einer direkt in die Assimilationskammer reichenden Meßzelle durch Druckschwankungen, Turbulenzänderungen, Lichteinflüsse ${ }^{2}$ etc. keine ungestörten Messungen möglich waren, wurde von der eigentlichen Assimilationskammer ein gesondertes Elektrodengefäß abgesetzt (1).

Eine eigens entwickelte Ganzglas-Zentrifugalpumpe in Form eines dicht unter der Meßßzelle rotierenden Ringmagneten (etwa $2000 \mathrm{U} / \mathrm{min}$ ), angetrieben durch einen regelbaren kleinen Magnetrührer (30) sorgt für eine gleichmäßige, ruhige und kräftige Umwälzung (etwa $0,31 / \mathrm{min}$ ).

Es zeigten sich keine elektrischen Störeffekte durch das starke, rotierende Magnetfeld. Zusammen mit einem in der Assimilationszelle unterhalb des Objektträgers laufenden Rührmagneten (angetrieben durch einen CENCO-Magnetrührer [30], etwa $250 \mathrm{U} / \mathrm{min}$ ) sorgt die Pumpe für eine rasche und kräftige Durchmischung des Versuchswassers. Die Verzögerung der Messung durch die räumliche Abtrennung der Elektrode von der Assimilationszelle konnte dabei völlig vernachlässigt werden.

\section{AUFBAU UND INBETRIEBNAHME DER MESSZELLE}

In einen Mantel aus PVC (Abb. $5 \mathrm{C}$ [16]) ist ein Träger (15) aus dem gleichen Material eingeschraubt, der die Anode (14) aus Feinsilberdraht $(\varnothing=1,0 \mathrm{~mm}$ ) trägt.

${ }^{2}$ Nach mündlicher Mitteilung von JANKowsKY (Zoologisches Institut der Universität Kiel) können besonders bei Verwendung von KCl-Lösungen als Elektrolyt erhebliche Störeffekte durch Licht auftreten. Wir selbst konnten solche nur nach längerem Gebrauch der Elektrode feststellen. 
Die Pt-Elektrode (13) ( $\varnothing 8,0 \mathrm{~mm}$; Stärke 2,0 mm) mit einer angelöteten Zuleitung (22) aus $\mathrm{Ag}$-Draht ist mit einem 2-Komponenten-Kleber in die Stirnseite des Trägers eingeklebt. Da sich bei längerem Gebrauch an dieser Stelle oft feine Haarrisse zwischen PVC und Kleber bildeten, die zu starken elektrischen Störungen führten (Elementbildung zwischen Lötzinn und Ag), wurde in einer weiterentwickelten Ausführung der PVC-Träger durch einen Glasstempel ersetzt, in den eine $2 \mathrm{~mm}$ starke Pt-Scheibe eingeschmolzen war (Zuleitung über einen Pt-Draht und Hg-Kontakt [24]). Sämtliche Glasarbeiten wurden von der Firma Gohla, Kiel, ausgeführt,

Die Dichtung an der Verschraubung erfolgt mittels einer PVC-Kontermutter (23) und O-Ring (19). Seitlich in dem Mantel befindet sich ein Schraubventil (18) zur Entlüftung und zum Druckausgleich.

Als Elektrolyt wird eine 0,2 n KOH-Lösung verwendet (Grasshoff 1962b), die vor dem Einfüllen sorgfältig in einem Wasserstrahlvakuum zu entgasen ist. Vor der Inbetriebnahme der Meßzelle muß die Pt-Kathode sorgfältig poliert und gereinigt werden. Erst danach kann sie mit einer $12 \mu$ starken Cellophan-Folie überzogen werden, die zuvor in aqua destillata eingeweicht wird. Eine $20 \mu$ starke Lupolen-Folie (BASF) - mittels eines 0-Ringes am konischen Vorderteil des Mantels befestigt schlicßt den Elektrolytraum vom Außenmedium ab.

Die genau in den Schliff des Elektrodengefäßes passende Konushïlse (17) wird vor dem Aufsetzen auf den Vorderteil des Mantels innen mit Wollfett eingestrichen, um eine clektrische Isolierung des Elektrolytraumes gegen das Außenmedium zu sichern.

\section{TEMPI:RATURREGEI.UNG}

Wie weiter oben ausgeführt, ist der Depolarisationsstrom diffusionskontrolliert und damit in weitem Maße temperaturabhängig. Es muß daher ganz besonders auf die Einhaltung einer möglichst konstanten Arbeitstemperatur Wert gelegt werden. Assimilationskammer und Elektrodengefäß sind deshalb völlig mit einem aus Plexiglas gefertigten Kühlmantel umgeben. In diesen wird aus einem gut isolierten, 15 I fassenden Thermostatenbad (Abb. $5 \mathrm{D}$ [31]) ständig ein kräftiger Wasserstrom geleitet. Ebenso wird der hohle Verschlußdeckel der Assimilationskammer von dem Kuhlmittel durchströmt.

Die Temperaturregelung erfolgte mittels eines in das Bad eingehängten Tauchthermostaten (Braun-Melsungen; Regelgenauigkeit $\pm 0,01^{\circ} \mathrm{C}$ ). Aus einem Kühlaggregat wurde zur Gegenkühlung (Abb. $5 \mathrm{C}$ [33]) auf $5^{\circ}$ bis $10^{\circ} \mathrm{C}$ temperiertes Kühlmittel durch die Kühlschlange des Thermostaten gepumpt. Sämtliche Zuleitungen waren mit Schaumgummischlauch isoliert.

Auf Grund des relativ großen Badvolumens von 15 l, guter Isolierung, sorgfältig abgestimmter Gegenkühlung und rascher Umwälzung, lagen die temperaturbedingten Schwankungen unterhalb der Ablesegenauigkeit des MeRinstrumentes, betrugen also weniger als $\pm 0,05 \mathrm{mV}$. Das entspricht bei der verwendeten Elektrodenanordnung einer rechnerisch ermittelten Temperaturkonstanz von etwa $\pm 0,005^{\circ} \mathrm{C}$. 


\section{REGISTRIERUNG}

Als Meßinstrumente eignen sich möglichst niederohmige und empfindliche Ampèremeter (Nanoampèremeter). Da uns für die $\mathrm{pO}_{2}$-Messung kein entsprechend empfindliches Strommeßgerät zur Verfügung stand, wurde mit einem Kompensationsschreiber (Compcorder Model CC-03, TOA-Electronics Ltd., Tokio, Japan) der Spannungsabfall über $5 \mathrm{k} \Omega$ registriert. Die zugehörige Schaltung ist in Abbildung 4 dargestellt. Als Spannungsquelle für die $\mathrm{O}_{2}$-Elektrode diente ein $\mathrm{Ni}$-Cd-Akkumulator $(1,2 \mathrm{~V} ; 7,5 \mathrm{Ah})$.

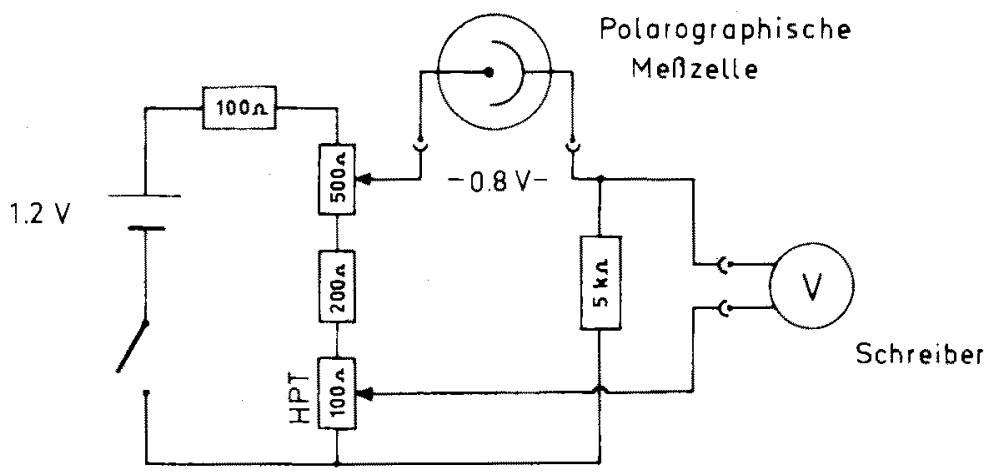

Abb. 4: Schaltung für Spannungsmessung

$\mathrm{Um}$ für jeden $\mathrm{pO}_{2}$ im empfindlichsten Bereich des Schreibers $(5 \mathrm{mV}$ ) messen zu können, war es mittels eines Präzisionspotentiometers möglich, den Nullpunkt entsprechend zu verschieben.

\section{LICHTQUELLE}

Als künstliche Lichtquelle (Abb. 5 D [34]) wurde eine Photoleuchte (OsramNitraphot $B R, 250 \mathrm{~V} / 500 \mathrm{~W})$ verwendet. Bei einem Abstand von $47 \mathrm{~cm}$ vom Untersuchungsobjekt betrug die Beleuchtungsstärke etwa $10000 \mathrm{Lux}$.

Zur Schwächung der intensiven Wärmestrahlung war zwischen Lampe und Assimilationskammer eine $10 \mathrm{~cm}$ dicke Kühlwasserschicht angebracht worden (Abb. 5 D [35]).

\section{EICHUNG}

Die Eichung der Elektrode erfolgte in der Assimilationskammer bei Versuchsbedingungen. Nach Einstellen einer konstanten Anzeige wurde das Versuchswasser über den Ablauf in 50-ml-Flaschen abgelassen und der Sauerstoff chemisch-analytisch nach WinkLer bestimmt. Durch Einleiten von $\mathrm{N}_{2}$ konnte der $\mathrm{O}_{2}$-Gehalt des Wassers in Stufen herabgesetzt, beziehungsweise durch Einleiten von $\mathrm{O}_{2}$ heraufgesetzt werden. 
Bei einwandfreier Funktion ergibt sich stets eine lineare Beziehung zwischen $\mathrm{O}_{2}-$ Gehalt und Diffusionsstrom in dem untersuchten Bereich zwischen $\mathrm{O}$ und $10 \mathrm{mg} \mathrm{O}_{2} / \mathrm{l}$. Außerdem schneidet die Eichkurve den O-Punkt (Abb. 3). Ist die lineare Beziehung einmal festgestellt, genügt es, einen sicheren Eichpunkt zu bestimmen. Bei den laufenden Kontrollmessungen zur Überprüfung der Drift der Elektrode begnügten wir uns daher meistens mit der Messung des Wertes für Luft bei $15^{\circ} \mathrm{C}$ oder für etwa luftgesättigtes Seewasser $\left(15 \%\right.$ S, $15^{\circ} \mathrm{C}$ ) (siehe auch Tö́t 1958, Kanwisher 1959). Die absolute Genauigkeit der Eichung wird durch die Genauigkeit der Winkler-Methode begrenzt (Grasshoff 1962a: Standardabweichung der Winkler-Methode $\pm 0,62 \%$ vom Mittelwert).

\section{LEISTUNGSFAHIGKEIT DER METHODE UND FEHLERMOGLICHKEITEN}

Die relative Meßgenauigkeit wird im wesentlichen durch das Meßinstrument bestimmt. Bei $0,1 \mathrm{mV}$ Ablesegenauigkeit lassen sich mit unserer Meßanordnung und bei unseren Versuchsbedingungen $\left(15^{\circ} \mathrm{C}, 15 \% \mathrm{~S}\right)$ Sauerstoffänderungen von etwa $0,0035 \mathrm{mg} \mathrm{O}$ erfassen.

Die Bestimmung der Temperaturabhängigkeit des Diffusionsstromes ergab für $1^{0} \mathrm{C}$ Temperaturerhöhung eine Erhöhung der Anzeige um 5 bis $7 \%$. Sie kann bei der von uns erreichten Temperaturkonstanz $\left(t \pm 0,005^{\circ} \mathrm{C}\right.$ ) vernachlässigt werden.

$\mathrm{Zu}$ den der Methode der elektrochemischen $\mathrm{O}_{2}$-Bestimmung anhaftenden Fehlern gehört die Drift der Elektrode (Tönt 1958, Grasshome 1962b). Die relative Schwankung über Tage wird von Grasshorf mit $0,8 \%$ angegeben. Uber einen Zeitraum von 4 Wochen betrug bei unseren eigenen Messungen die maximale Abweichung vom Mittelwert etwa $2 \%$. Für die Einzelmessung ist die Drift zu vernachlässigen.

Ebenso kann der $\mathrm{O}_{2}$-Eigenverbrauch der Elektrode bei der Größe des verwendeten Wasservolumens (307 ml) unberücksichtigt bleiben (GLHCHMANN \& LÜBBERS 1960).

Verfälschungen der Anzeige auf Grund der Einstellzeit (etwa 14 bis $16 \mathrm{sec}$ bis zum Erreichen von $90 \%$ der $\mathrm{O}_{2}$ - Anderung) sind nur bei sprunghaften $\mathrm{pO}$-Anderungen von Bedeutung (Grasshofr 1962b). Weitere ausführliche Angaben zu den systematischen Fehlern der elektrochemischen Sauerstoffbestimmung finden sich bei Tönt (1958), Gleichmann \& Lübbers (1960), Grasshoff (1962b), Koyama (1965). Im folgenden sollen nur die Fehlermöglichkeiten für die von uns verwendete speziellc Versuchsanordnung erörtert werden.

Die Brauchbarkeit und Exaktheit der Methode ist in weitem Maße von der Einhaltung der Versuchstemperatur abhängig. Abweichungen können zu Verfälschungen der $\mathrm{O}_{2}-\mathrm{Messungen} \mathrm{führen.} \mathrm{So} \mathrm{kommt} \mathrm{es} \mathrm{besonders} \mathrm{bei} \mathrm{größeren} \mathrm{Unterschieden} \mathrm{zwischen}$ der Raumtemperatur und der des Versuchswassers während des Offnens der Assimilationskammer und beim Einlegen des Untersuchungsobjektes zu kurzfristigen Veränderungen der Anzeige um etwa $\pm 0,2$ bis $0,5 \mathrm{mV}$. Dies ist in erster Linie auf eine geringfügige Erhöhung oder Erniedrigung der Versuchstemperatur zurückzuführen. Die Rückregelung erfolgt in 1 bis 2 min. Dieser Fehler wird weitgehend vermieden, wenn die Temperaturen des Versuchswassers, des Raumes und besonders des Untersuchungsmaterials möglichst wenig voneinander abweichen. 
Eine weitere Ursache für plötzliche Veränderungen der Anzeige beim Öfnen der Assimilationszelle kann die zusätzliche Lösung von Luftsauerstoff im beziehungsweise das Herausdiffundieren von $\mathrm{O}_{2}$ aus dem Versuchswasser sein. Da im allgemeinen jedoch im Bereich der Luftsätrigungswerte gearbeitet wurde, blieb dieser Fehler nur sehr gering. Außerdem hat er keinen Einfluß auf den Meßvorgang.
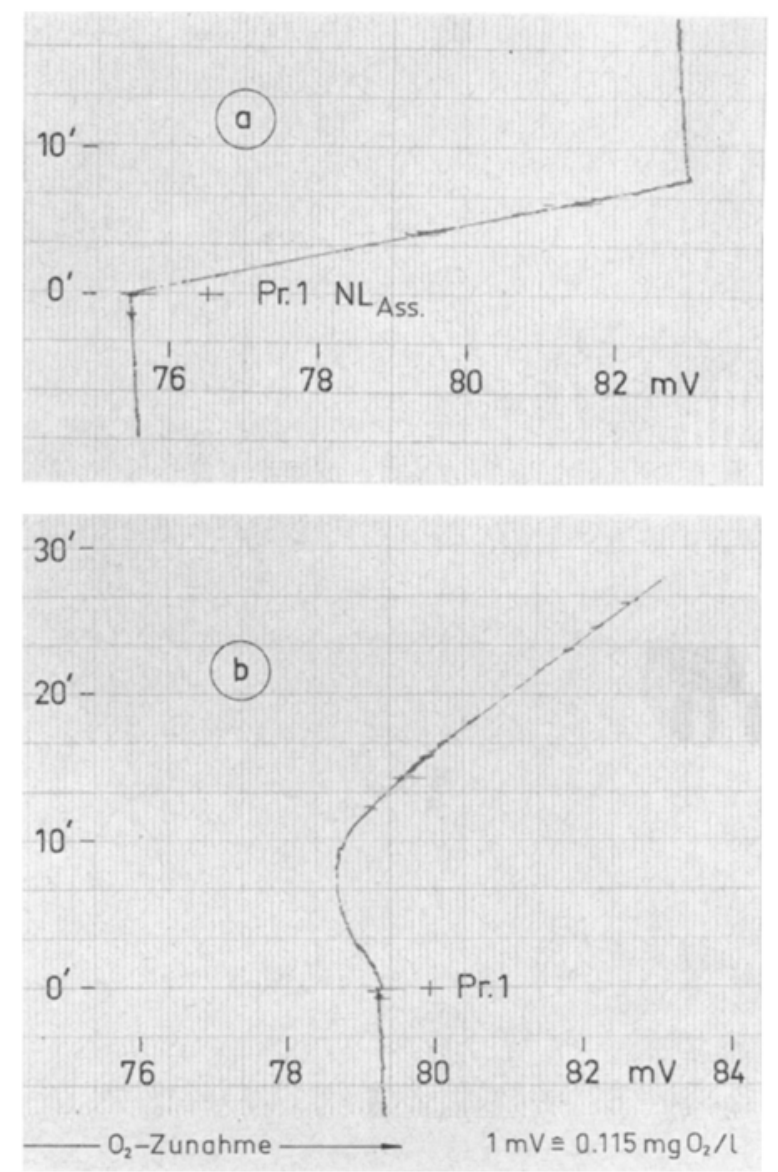

Abb. 6: Originalregistrierung der Assimilation von Fucus vesiculosus $\left(10000 \mathrm{~lx}, 15^{\circ} \mathrm{C}\right.$, Ostseewasser von $15 \%$ S). (a) Assimilationsgrundleistung, (b) Assimilation beim Wiedereinquellen nach Austrocknung auf $25 \%$ des Sättigungsgewichtes. Ordinate: Zeit in Minuten; Abzisse: $\mathrm{O}_{2}$-Konzentrationsänderung in $\mathrm{mV}$

\section{ANWENDUNGSBEISPIELE UND DISKUSSION DES VERFAHRENS}

Bei den von uns durchgeführten Untersuchungen zur Austrodknungs- und Temperaturresistenz mariner Algen wurde besonders auf den Verlauf der Reaktivierung von Assimilation und Atmung beim Wiedereinquellen Wert gelegt. Die zuvor verschieden 
stark entquollenen Algenproben wurden zu diesem Zweck nach einer kurzen Adaptionszeit an die bei der Messung herrschenden Licht- und Temperaturverhältnisse direkt in die Assimilationskammer überführt.

In Abbildung 6 sind Originalregistrierungen der Assimilations-Grundleistung (NL Ass.) von Fucus vesiculosus L. und des Assimilationsverlaufes beim Wiedereinquellen nach vorheriger Austrocknung auf $25 \%$ des Sättigungsgewichtes wiedergegeben. Die Messungen erfolgten bei $10000 \mathrm{~lx}$ und $15^{\circ} \mathrm{C}$ in Ostseewasser von $15 \%$ S.

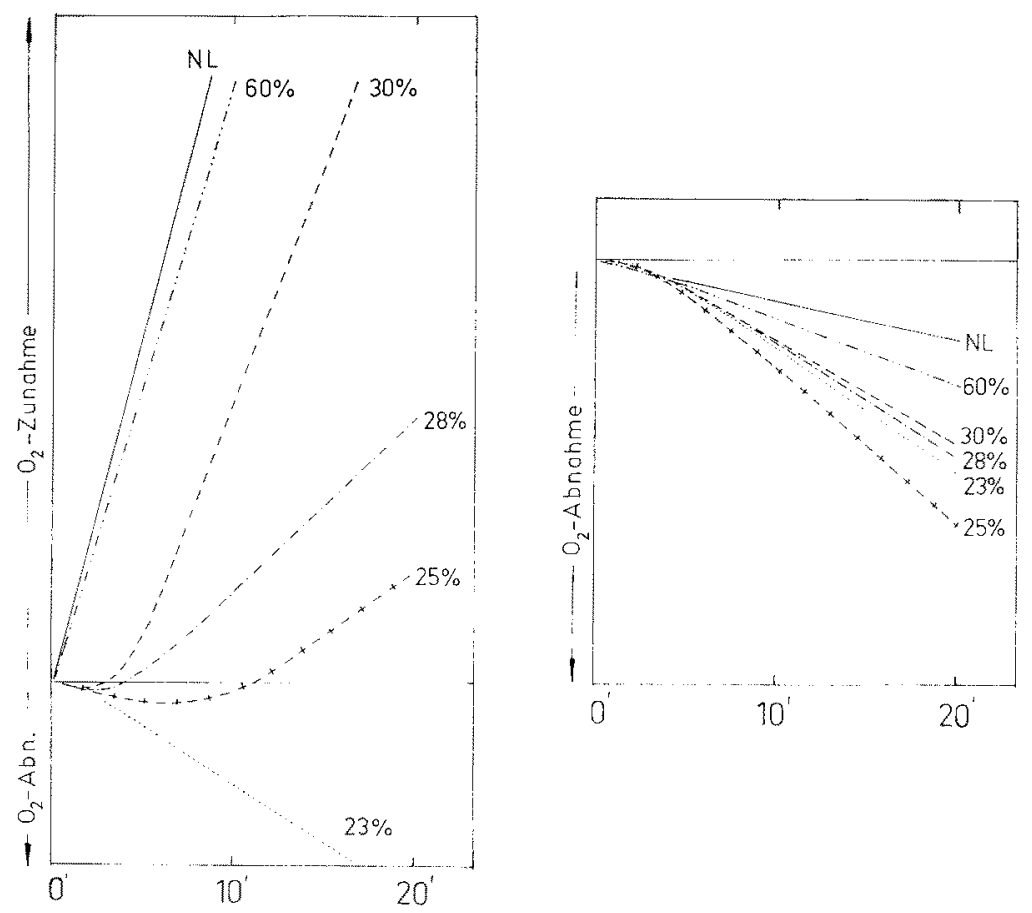

Abb. 7: Assimilation und Atmung beim Wiedereinquellen nach verschieden starker Entquellung (in "1/0 des Sättigungsgewichts) bei $15^{\circ} \mathrm{C}$ in Ostscewasser von $15 \%$ S). Links: Assimilation $(10000 \mathrm{~lx})$; rechts: Dunkelatmung. Ordinate: Oz-Konzentrationsänderung; Abzisse: Quellzcit in Minuten; NL: mittlere Grundleistung

Die Geradlinigkeit des Kurvenverlaufs bei der Bestimmung der Grundleistung beweist, daß die geringfügigen $\mathrm{O}_{2}$-Konzentrationsänderungen ( 5 bis $10 \%$ des Sättigungswertes) während der 10 - bis 30 minütigen Messungen ohne Einfluß auf die Assimilation oder Atmung bleiben. Die Steigung der Kurven ist ein Maß für die Gaswechselintensität, die in ihren absoluten Werten bei bekannter Materialmenge leicht anhand der Eichkurven ermittelt werden können.

Bei vergleichenden Untersuchungen an verschiedenem Material wurden die Originalregistrierungen auf einheitliches Sättigungsgewicht oder mittlere Grundleistung ( $=$ NL) korrigiert. Abbildung 7 zeigt die Zeitkurven der Assimilation und 
Atmung für Fucus vesiculosus nach verschieden starker Entquellung. Während der Atmungsmessungen wurde die Assimilationskammer mit einem schwarzen Tuch abgedeckt.

Die Ergebnisse zeigen deutlich eine mit dem Austrocknungsgrad zunehmende anfängliche Depression der Assimilation, die nach starker Entquellung (23\% des Sättigungsgewichts) erst nach Stunden überwunden wird. Die Atmung beim Wiedereinquellen erfährt dagegen mit zunehmenden Wasserverlusten eine Steigerung. Diese geht nach starker Entquellung $(23 \%)$ wieder zurïck. Nach extremer Austrodknung kann der Sauerstoff verbrauch sogar unter der Normalleistung liegen.

Der Vorteil der Meßanordnung besteht in erster Linie in der Möglichkeit, den Verlauf geringer Sauerstoffänderungen unmittelbar nach dem Einbringen des Untersuchungsmaterials in die Apparatur kontinuierlich zu messen. Die Konstruktion der Assimilationskammer gestattet es, den $\mathrm{O}_{2}$-Gaswechsel verschiedenartigster pflanzlicher oder tierischer Objekte zu verfolgen. Daneben lassen sich ohne Schwierigkeiten die Versuchsbedingungen (Licht, Temperatur, Medium) in weiten Grenzen variieren.

\section{ZUSAMMENFASSUNG}

1. Eine Apparatur zur kontinuierlichen Messung von Assimilation und Atmung mariner Organismen nach dem Prinzip der elektrochemischen Sauerstoffbestimmung wird beschrieben.

2. Die Or-Messung erfolgt in einem geschlossenen System mittels einer stabilisierten, membranüberzogenen Pt-Elektrode (CLARK-Prinzip) mit folgenden Eigenschaften: Die Einstellzeit auf $90 \%$ des Endwertes beträgt 14 bis $16 \mathrm{sec}$; die Drift liegt über Tage bei $0,8 \%$, über Wochen bei $2 \%$. Im Bereich von 0 bis $10 \mathrm{mg} \mathrm{O}_{2} / 1$ ist die Beziehung $\mathrm{zwischen}$ Depolarisationsstrom und $\mathrm{O}_{2}$-Konzentration linear. Die Eichkurve schneidet den Nullpunkt.

3. Mit der beschriebenen Anordnung können Sauerstoffänderungen von $0,0035 \mathrm{mg} \mathrm{O}$ erfaßt werden. Es werden Beispiele für die Anwendung von Assimilations- und Atmungsmessungen an der Braunalge Fucus vesiculosus L. angeführt.

Die Untersuchungen wurden im Institut für Meereskunde der Universität Kiel, Botanische Abteilung, auf Anregung und unter der Leitung von Herrn Prof. Dr. F. GEssNer durchgeführt. Die Arbeiten wurden aus Mitteln der Deutschen Forschungsgemeinschaft finanziert.

\section{ZITIERTE LITERATUR}

АмвӥнL, H., 1958. Die praktische Anwendung der elektrochemischen Sauerstoffbestimmung im Wasser. Schweiz. Z. Hydrol. 20, 341-359.

BARBEY, K., 1953. Sauerstoffversorgung und Sauerstoffverbrauch des Froschherzens in Ruhe. Kiel, Med. Diss. v. 24. 2. 1954, 25 Bl.

Clark, L. C., Wolf, R., Granger, D. \& Taylor, A., 1953. Continuous recording of blood oxygen tensions by polarography. J. appl. Physiol. 6, 189-193.

Egle, K., 1960. Landpflanzen. In: Handbuch der Pflanzenphysiologie. Hrsg. von Wilhelm Ruhland (u. a.). Springer, Berlin, 5, 115-163. 
Føxn, E., 1955. Continuous oxygen recording in sea water. FiskDir. Skr. (Ser. Havunders.) $11(3), 1-8$.

Gleichmann, U. \& Lübibrs, D. W., 1960. Die Messung der Sauerstoffdrucke in Gasen und Flüssigkeiten mit der Platinelektrode unter besonderer Berücksichtigung der Messung im Blut. Pfügers Arch. ges. Physiol. 271, 431-455.

GRAsshoff, K., 1962a. Untersudungen über die Sauerstoffbestimmung im Meerwasser. T. 1. Kieler Meeresforsch. 18, 42-50.

- 1962b. Untersuchungen über die Sauerstoffbestimmung im Meerwasser. T. 2. Kieler Meeresforsch. 18, 151-160.

- 1963. Untersuchungen über die Sauerstoffbestimmungen im Meerwasser. Kieler Meeresforsch. 19, 8-15.

HeYrovskx, J., 1922. Elektrolysa se rtutovou kapkovou kathodou. Chemicke Listy 16, 256.

- 1948. Polarographisches Praktikum. Springer, Berlin, $118 \mathrm{pp}$.

Kanwisher, J., 1959. Polarographic oxygen electrode. Limnol. Oceanogr. 4, 210-217.

Koyama, T. \& Brecht, K., 1965. Ein Beitrag zur Stabilisierung der Pt-Elektrode und ihre Anwendung bei gleichzeitiger Bestimmung des Sauerstoffverbrauchs und der mechanischen Aktivität des Muskels. Pfiügers Arch, ges. Physiol. 286, 181-188.

Müllek, J., 1958. Uber die Verwendung von Magnos-Sauerstoffschreibern für Gaswechselregistrierungen in der Biologie. Ber. dt. bot. Ges. 71, 205-223.

OHLL, W., 1953. Dic chemische und elektrochemische Bestimmung des molekular gelösten Sauerstoffs der Binnengewässer. Mitt, int. Verein. theor. angew. Limnol. 3. 1-44.

Rooth, G., Sjöstedt, S. \& Caligara, F., 1960. Oxygen tension of the blood in the umbilical cord and the intervillous space. Archs Dis. Childh. 35, 529-533. (Zit. nach Gleichmann, U. \& LÜBBERS, D. W., 1960.)

Seviringhaus, J. W. \& Bradey, A. F., 1958 . Flectrodes for blood pO,- and pCOz-determination. J appl. Pbysiol. 13,515-520.

Simonis, W., 1960. Wasserpflanzen. In: Handbuch der Pflanzenphysiologie. Hrsg. von Wilhelm Ruhland (u. a.). Springer, Berlin, 5, 164-181.

Tönt, F., 1929. Die laufende Anzeige des Gehaltes an gelösten Sauerstoff sowie des durch Natronlauge bewirken Rostschutzes durch Strommessung. Z. Vor. at. Zuckind. (1929), $680-694$.

- 1952. Die Messung der kathodischen Sauerstoffredukrion durch geeignete Modellelemente. Z. Elektrochem. 56, 163-169.

- 1958. Hilektrochemische Sauerstoffmessungen. De Gruyter, Berlin, 212 pp.

Winki:LmanN, D., 1956. Untersuchungen über das elektrochemische Verhalten von O., HoO,$\mathrm{H}_{2} \mathrm{O}$ an blankem und platiniertem Platin. Z. Elektrochem. 60,731.

Winkter, L. W., 1888. Die Bestimmung des im Wasser gelosten Sauerstoffs, Ber. dt. chem. Ges. 21, 2843-2854. 